FERMILAB-PUB-04-140-T

hep-ph/0408070

\title{
Untangling
}

\section{CP Violation and the Mass Hierarchy in Long Baseline Experiments}

\author{
Olga Mena and Stephen Parke \\ Theoretical Physics Department, \\ Fermi National Accelerator Laboratory \\ P.O.Box 500, Batavia, IL 60510, USA \\ omena@fnal.gov parke@fnal.gov
}

(Dated: July 31, 2004)

\begin{abstract}
In the overlap region, for the normal and inverted hierarchies, of the neutrino-antineutrino biprobability space for $\nu_{\mu} \rightarrow \nu_{e}$ appearance, we derive a simple identity between the solutions in the $\left(\sin ^{2} 2 \theta_{13}, \sin \delta\right)$ plane for the different hierarchies. The parameter $\sin ^{2} 2 \theta_{13}$ sets the scale of the $\nu_{\mu} \rightarrow \nu_{e}$ appearance probabilities at the atmospheric $\delta m_{\text {atm }}^{2} \approx 2.4 \times 10^{-3} \mathrm{eV}^{2}$ whereas $\sin \delta$ controls the amount of $\mathrm{CP}$ violation in the lepton sector. The identity between the solutions is that the difference in the values of $\sin \delta$ for the two hierarchies equals twice the value of $\sqrt{\sin ^{2} 2 \theta_{13}}$ divided by the critical value of $\sqrt{\sin ^{2} 2 \theta_{13}}$. We apply this identity to the two proposed long baseline experiments, $\mathrm{T} 2 \mathrm{~K}$ and $\mathrm{NO} \nu \mathrm{A}$, and we show how it can be used to provide a simple understanding of when and why fake solutions are excluded when two or more experiments are combined. This identity demonstrates the true complimentarity of $\mathrm{T} 2 \mathrm{~K}$ and $\mathrm{NO} \nu \mathrm{A}$.
\end{abstract}

PACS numbers: $14.60 \mathrm{Pq}$

Typeset by REVTEX 
With the possibility of the first measurement of $\theta_{13}$ being made by a 1 to $2 \mathrm{~km}$ baseline reactor experiment [1], the long baseline $\nu_{e}$ appearance experiments, T2K [2] and $\mathrm{NO} \nu \mathrm{A}[3]$, need to adjust their focus to emphasize other physics topics. The most important of these questions is the form of the mass hierarchy, normal $\left(\delta m_{31}^{2}>0\right)$ versus inverted $\left(\delta m_{31}^{2}<0\right)$, and whether or not leptonic CP violation occurs, $(\sin \delta \neq 0)$. Matter effects [4] entangle these questions [5]. Suppose $P\left(\nu_{\mu} \rightarrow \nu_{e}\right)<P\left(\bar{\nu}_{\mu} \rightarrow \bar{\nu}_{e}\right)$, then in vacuum this implies CP violation, however in matter this implies $\mathrm{CP}$ violation only for the normal hierarchy but not necessarily for the inverted hierarchy. The purpose of this paper is to demonstrate that there is a simple way to understand this entanglement and to use this understanding to untangle the mass hierarchy question from whether or not leptonic CP violation occurs.

The outline of this paper is as follows: Along the diagonal of the $\nu_{\mu} \rightarrow \nu_{e}$ bi-probability diagram, see Figs. 1 and 2, we solve for $\theta_{13}$ and $\delta$ exactly, i.e. we have imposed the constraint $P\left(\nu_{\mu} \rightarrow \nu_{e}\right)=P\left(\bar{\nu}_{\mu} \rightarrow \bar{\nu}_{e}\right)$. There are four such solutions ${ }^{1}$, two for the normal hierarchy [8] and two for the inverted hierarchy [9, 10]. With these solutions we derive an identity connecting the difference in the mean values of $\sin \delta$ (the $\mathrm{CP}$ violating parameter) for the two hierarchies to the mean values of $\theta_{13}$ for these solutions. Although this identity is derived along the diagonal, in an Appendix we present the corrections to this identity off the diagonal using the approximate solutions derived in Ref.[11]. We then apply this identity to the proposed long baseline experiments $\mathrm{T} 2 \mathrm{~K}$ and $\mathrm{NO} \nu \mathrm{A}$. We show that the fake solutions for these two experiments occur in different parts of parameter space and therefore they can be excluded with sufficient statistics 12]. The identity relating the two mean values of $\sin \delta$, one for the normal hierarchy and one for inverted hierarchy is the new result of this paper and it provides a simple physics understanding of when various fake solutions are excluded when experiments are combined.

The $\nu_{\mu} \rightarrow \nu_{e}$ appearance probabilities in long baseline neutrino oscillation experiments, assuming the normal mass hierarchy, can be written as [8]

$$
\begin{aligned}
& P\left(\nu_{\mu} \rightarrow \nu_{e}\right)=X_{+} \theta^{2}+Y_{+} \theta \cos \left(\Delta_{13}+\delta\right)+P_{\odot} \\
& \bar{P}\left(\bar{\nu}_{\mu} \rightarrow \bar{\nu}_{e}\right)=X_{-} \theta^{2}-Y_{-} \theta \cos \left(\Delta_{13}-\delta\right)+P_{\odot}
\end{aligned}
$$

\footnotetext{
${ }^{1}$ We assume $\theta_{23}=\pi / 4[\underline{6}$, 7] initially and discuss generalizations later.
} 
In the last expressions, $\theta=\sin \theta_{13}$ and the coefficients $X_{ \pm}$and $Y_{ \pm}$are determined by

$$
\begin{aligned}
& X_{ \pm}=4 s_{23}^{2}\left\{\frac{\Delta_{13} \sin \left(a L \mp \Delta_{13}\right)}{\left(a L \mp \Delta_{13}\right)}\right\}^{2}, \\
& Y_{ \pm}= \pm 2 \sqrt{X_{ \pm} P_{\odot}}= \pm 8 c_{12} s_{12} c_{23} s_{23}\left\{\frac{\Delta_{13} \sin \left(a L \mp \Delta_{13}\right)}{\left(a L \mp \Delta_{13}\right)}\right\}\left\{\frac{\Delta_{12} \sin (a L)}{a L}\right\} \\
& P_{\odot}=c_{23}^{2} \sin ^{2} 2 \theta_{12}\left\{\frac{\Delta_{12} \sin (a L)}{a L}\right\}^{2}
\end{aligned}
$$

where $\Delta_{i j} \equiv\left|\Delta m_{i j}^{2}\right| L / 4 E$ and $a=G_{F} N_{e} / \sqrt{2}$ denotes the index of refraction in matter with $G_{F}$ being the Fermi constant and $N_{e}$ a constant electron number density in the earth. Obviously from the above definitions, $X_{ \pm}$and $Y_{ \pm}$satisfy the identity

$$
\frac{Y_{+}}{\sqrt{X_{+}}}=-\frac{Y_{-}}{\sqrt{X_{-}}}
$$

which is used extensively throughout this paper.

To solve equations Eqn. 1] exactly with the constraint $P=\bar{P}$, i.e. along the diagonal of the bi-probability diagram, we use the ansatz

$$
\theta=\theta_{c}(\sin \delta-\beta \cos \delta)
$$

where

$$
\theta_{c}=\frac{Y_{+}}{\sqrt{X_{+}}} \frac{\sin \Delta_{13}}{\left(\sqrt{X_{+}}-\sqrt{X_{-}}\right)} \quad \text { and } \quad \beta=\left(\frac{\sqrt{X_{+}}-\sqrt{X_{-}}}{\sqrt{X_{+}}+\sqrt{X_{-}}}\right) \frac{\cos \Delta_{13}}{\sin \Delta_{13}}
$$

Then

$$
P=\bar{P}=\sqrt{X_{+}} \sqrt{X_{-}} \theta_{c}^{2}\left(\sin ^{2} \delta-\beta^{2} \cos ^{2} \delta\right)+P_{\odot}
$$

$\mathrm{P}$ has a maximum when $\sin \delta=1, \theta=\theta_{c}$ and $P_{c}=\sqrt{X_{+}} \sqrt{X_{-}} \theta_{c}^{2}+P_{\odot}$. We call these values the critical values of $\mathrm{P}$ and $\theta$. There are no solutions along the diagonal for values of $\mathrm{P}$ larger than $P_{c}$.

Using this critical value of $\mathrm{P}$ to normalize the probabilities, we can solve for $\delta$. Thus the exact solutions, labeled 1 and 2, for the normal hierarchy, are

$$
\begin{aligned}
& \theta_{1}=\theta_{c}\left(s_{p}-\beta c_{p}\right), \quad \sin \delta_{1}=s_{p} \quad \text { and } \cos \delta_{1}=c_{p} \\
& \theta_{2}=\theta_{c}\left(s_{p}+\beta c_{p}\right), \quad \sin \delta_{2}=s_{p} \quad \text { and } \cos \delta_{2}=-c_{p}
\end{aligned}
$$

where

$$
s_{p} \equiv+\sqrt{\frac{\left(P-P_{\odot}\right) /\left(P_{c}-P_{\odot}\right)+\beta^{2}}{1+\beta^{2}}} \quad \text { and } \quad c_{p} \equiv+\sqrt{\frac{1-\left(P-P_{\odot}\right) /\left(P_{c}-P_{\odot}\right)}{1+\beta^{2}}} .
$$


Along the diagonal the two solutions for the $\mathrm{CP}$ violating parameter, $\sin \delta$, are identical, $\sin \delta_{1}=\sin \delta_{2}$.

For the inverted hierarchy, the $\nu_{\mu} \rightarrow \nu_{e}$ appearance probabilities are

$$
\begin{aligned}
& P\left(\nu_{\mu} \rightarrow \nu_{e}\right)=X_{-} \theta^{2}+Y_{-} \theta \cos \left(\Delta_{13}-\delta\right)+P_{\odot} \\
& \bar{P}\left(\bar{\nu}_{\mu} \rightarrow \bar{\nu}_{e}\right)=X_{+} \theta^{2}-Y_{+} \theta \cos \left(\Delta_{13}+\delta\right)+P_{\odot} .
\end{aligned}
$$

These equations are identical to the equations for the normal hierarchy when we use the constraint $P=\bar{P}$ and replace $\delta$ with $\delta+\pi$, then, the solutions are

$$
\begin{aligned}
& \theta_{3}=\theta_{c}\left(s_{p}-\beta c_{p}\right), \quad \sin \delta_{3}=-s_{p} \quad \text { and } \cos \delta_{3}=-c_{p} \\
& \theta_{4}=\theta_{c}\left(s_{p}+\beta c_{p}\right), \quad \sin \delta_{4}=-s_{p} \quad \text { and } \cos \delta_{4}=c_{p} .
\end{aligned}
$$

Note that $\theta_{3}=\theta_{1}$ with $\delta_{3}=\pi+\delta_{1}$ and $\theta_{4}=\theta_{2}$ with $\delta_{4}=\pi+\delta_{2}$.

With these solutions in hand it is simple to derive the principal result of this paper,

$$
\langle\sin \delta\rangle_{+}-\langle\sin \delta\rangle_{-}=2\langle\theta\rangle / \theta_{c}
$$

where $\langle\sin \delta\rangle_{+(-)}=\left(\sin \delta_{1(3)}+\sin \delta_{2(4)}\right) / 2$, the mean values of $\sin \delta$ for each hierarchy, and $\langle\theta\rangle=\left(\theta_{1}+\theta_{2}+\theta_{3}+\theta_{4}\right) / 4$, the mean value of $\theta$ for both hierarchies. For $P=\bar{P}$ there are many ways to write this expression, however we write it in this way because with these variables it is accurate even if $P \neq \bar{P}$. In vacuum, $\theta_{c} \rightarrow \infty$ so that the values of $\sin \delta$ for the two hierarchies are identical.

The physical meaning of this result is clear, i.e the difference in the mean values of $\sin \delta$ (the $\mathrm{CP}$ violating parameter) between the mass hierarchies equals twice the mean value of $\theta$ divided by the critical value of $\theta$. Away from $P=\bar{P}$ it is well known that the difference between the solutions for $\sin \delta$ and $\theta$ within the same hierarchy are small 12$]$. This implies that the relationship given by Eqn. 111 is still useful and informative even when $P \neq \bar{P}$. In fact we have used the approximations of Ref.[11] to derive the corrections to this master equation and find that the corrections are of $\mathcal{O}\left(\beta^{2}\right)$. Also the difference between the solutions of $\sin \delta$ within a hierarchy are of $\mathcal{O}(\beta)$, see the Appendix. For the currently proposed experiments $\beta$ is less than or of order 0.1 so the corrections to Eqn. [11] are no larger than a few percent. In a follow up paper, we will explore in more detail the accuracy of this relationship throughout the whole overlap region. 
The proposed long baseline, off-axis experiments are T2K and No $\nu \mathrm{A}$. T2K utilizes a steerable neutrino beam from JHF and SuperKamiokande and/or HyperKamiokande as the far detector. The mean energy of the neutrino beam will be tuned to be at vacuum oscillation maximum, $\Delta_{13}=\frac{\pi}{2}$, which implies a $\left\langle E_{\nu}\right\rangle=0.6 \mathrm{GeV}$ at the baseline of 295 $\mathrm{km}$ using $\left|\delta m_{31}^{2}\right|=2.4 \times 10^{-3} \mathrm{eV}^{2}[\underline{6}]$. This is the $3^{o}$ off-axis beam. For this configuration the matter effects are small but not neglible [13] as can be seen from the separation of the allowed regions in the bi-probability diagram, Fig. 1, for this experiment. Applying our identity, Eqn.[1], to T2K, we find:

$$
\langle\sin \delta\rangle_{+}-\langle\sin \delta\rangle_{-}=0.47 \sqrt{\frac{\sin ^{2} 2 \theta_{13}}{0.05}} \quad \text { for } \mathrm{T} 2 \mathrm{~K}
$$

i.e. the difference between the true and fake solutions for the $\mathrm{CP}$ violating parameter $\sin \delta$ is $0.47(\approx \sqrt{2} / 3)$ at $\sin ^{2} 2 \theta_{13}=0.05$.

$\mathrm{NO} \nu \mathrm{A}$ proposes to use the Fermilab NuMI beam with a baseline of $810 \mathrm{~km}$ with a $50 \mathrm{kton}$ low $\mathrm{Z}$ detector which is $10 \mathrm{~km}$ off-axis resulting in a mean neutrino energy of $2.3 \mathrm{GeV}$. The $\mathrm{NO} \nu \mathrm{A}$ beam energy is about $30 \%$ above the vacuum oscillation maximum energy for this baseline. Matter effects are quite significant for $\mathrm{NO} \nu \mathrm{A}$ as can be seen from the bi-probability diagram, Fig 2. Applying our identity to $\mathrm{NO} \nu \mathrm{A}$ we find:

$$
\langle\sin \delta\rangle_{+}-\langle\sin \delta\rangle_{-}=1.41 \sqrt{\frac{\sin ^{2} 2 \theta_{13}}{0.05}} \quad \text { for } \mathrm{NO} \nu \mathrm{A}
$$

The difference between the true and fake solutions for the $\mathrm{CP}$ violating parameter $\sin \delta$ is $1.41(\approx \sqrt{2})$ at $\sin ^{2} 2 \theta_{13}=0.05$. The factor of 3 increase in the difference of the $\sin \delta$ 's compared to $\mathrm{T} 2 \mathrm{~K}$ is due to the coefficient in front of the square root which is proportional to (aL). The $\mathrm{NO} \nu \mathrm{A}$ detector is 2.75 times further away from the source than the T2K detector and the average density for the NOVA baseline is slightly higher than for the T2K baseline.

Combining the results from $\mathrm{T} 2 \mathrm{~K}$ and $\mathrm{NO} \nu \mathrm{A}$ we note that for the correct hierarchy and hence the true value of $\sin \delta$ the results should coincide within uncertainties

$$
\left|\langle\sin \delta\rangle_{t r u e}^{T 2 K}-\langle\sin \delta\rangle_{t r u e}^{N O \nu A}\right| \approx 0
$$

Whereas for the wrong hierarchy, the fake solutions of $\sin \delta$ are separated by

$$
\left|\langle\sin \delta\rangle_{\text {fake }}^{T 2 K}-\langle\sin \delta\rangle_{\text {fake }}^{N O \nu A}\right|=0.94 \sqrt{\frac{\sin ^{2} 2 \theta_{13}}{0.05}}
$$


This implies that if $\sin \delta$ can be measured with sufficient accuracy in both experiments not only could the hierarchy be determined but also the true value of the CP violating parameter $\sin \delta$ including in the overlap region. Even for $\sin ^{2} 2 \theta_{13}=0.01$, the separation of the fake solutions of $\sin \delta$ between experiments is 0.40 .

In Figs. 3 and 4 we have constructed the $\chi^{2}$ contours for both $\mathrm{T} 2 \mathrm{~K}$ and $\mathrm{NO} \nu \mathrm{A}$ assuming that the true solution is the normal hierarchy and that the values of $\left(\sin ^{2} 2 \theta_{13}, \delta\right)$ are $(0.05$, $\pi / 4)$ respectively. This point is near the middle of the overlap region in the bi-probability diagram for both $\mathrm{T} 2 \mathrm{~K}$ and $\mathrm{NO} \nu \mathrm{A}$ and it is one of the harder points to untangle the mass hierarchy and determine CP violation. Since T2K is operated at vacuum oscillation maximum there are only two allowed regions in the $\left(\sin ^{2} 2 \theta_{13}, \sin \delta\right)$ plane since this experiment is insensitive to the $\mathrm{CP}$ conserving quantity $\cos \delta . \mathrm{NO} \nu \mathrm{A}$ on the other hand is operated above oscillation maximum so this experiment is sensitive to the $\operatorname{sign}^{2}$ of $\cos \delta$. Therefore there are four solutions in $\left(\sin ^{2} 2 \theta_{13}, \sin \delta\right)$ plane. The approximate exposure that makes the ellipses in Figs. 3 and 4 the 68, 90 and 99\% C.L. contours is 5 years of both neutrino and anti-neutrino running with T2K operating at 0.75MW using HyperKamiokande as the

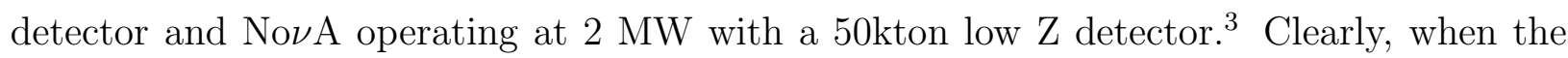
results of these two experiments are combined only the region near the true solution (normal hierarchy, $\sin ^{2} 2 \theta_{13} \approx 0.05$ and $\sin \delta \approx 0.7$ and $\cos \delta>0$ ), survives at more than $99 \%$ C.L.

If we allow $\theta_{23}$ to vary from $\pi / 4$ then the best variables to use are $\sqrt{2} \cos \theta_{23} \sin \delta$ and $2 \sin ^{2} \theta_{23} \sin ^{2} 2 \theta_{13}$. Using these variables we obtain the following identities:

$$
\sqrt{2} \cos \theta_{23}\langle\sin \delta\rangle_{+}-\sqrt{2} \cos \theta_{23}\langle\sin \delta\rangle_{-}=0.47 \sqrt{\frac{2 \sin ^{2} \theta_{23} \sin ^{2} 2 \theta_{13}}{0.05}} \text { for T2K }
$$

$$
\sqrt{2} \cos \theta_{23}\langle\sin \delta\rangle_{+}-\sqrt{2} \cos \theta_{23}\langle\sin \delta\rangle_{-}=1.41 \sqrt{\frac{2 \sin ^{2} \theta_{23} \sin ^{2} 2 \theta_{13}}{0.05}} \quad \text { for NO } \nu \mathrm{A}
$$

With these variables the figures equivalent to Figs. 3 and 4 but with $\sin ^{2} \theta_{23}$ varying between 0.35 and 0.65 (the allowed region from SuperKamiokande atmospheric neutrino results [6]) are almost identical except near the upper and lower boundary since the range of $\sqrt{2} \cos \theta_{23} \sin \delta$ for fixed $\sin ^{2} \theta_{23}$ is $\pm \sqrt{2} \cos \theta_{23}$, not \pm 1 as it is for $\theta_{23}=\pi / 4$.

\footnotetext{
${ }^{2}$ Given $\sin \delta$ one knows the magnitude of $\cos \delta$.

${ }^{3}$ We choose this combination so that the statistical uncertainty in $\sin \delta$ is approximately the same for both experiments.
} 
In summary we have derived a simple identity relating the solutions between the two hierarchies which allows one to compare the results from two or more long baseline experiments in a very straight forward manner. This identity was applied to the proposed T2K and $\mathrm{NO} \nu \mathrm{A}$ experiments and it demonstrates the true complimentary of these experiments in a simple, transparent fashion.

\section{ACKNOWLEDGEMENTS}

The authors wish to thank Hisakazu Minakata, Hiroshi Nunokawa, Takaaki Kajita and Mark Meisser for discussions. Fermilab is operated under DOE contract DE-AC0276CH03000. 


\section{APPENDIX}

For $P \neq \bar{P}$ we use the solutions, notation and approximations of [11]: (1 and 2 are labels for the solutions for the normal hierarchy and 3 and 4 for the inverted hierarchy.)

If we define

$$
\begin{aligned}
\langle\sin \delta\rangle_{+} & \equiv\left(\sin \delta_{1}+\sin \delta_{2}\right) / 2 \\
\langle\sin \delta\rangle_{-} & \equiv\left(\sin \delta_{3}+\sin \delta_{4}\right) / 2 \\
\langle\theta\rangle & \equiv\left(\theta_{1}+\theta_{2}+\theta_{3}+\theta_{4}\right) / 4 \\
\Omega \equiv 1+\beta^{2} & =1+\frac{\left(\sqrt{X_{+}}-\sqrt{X_{-}}\right)^{2} \cos ^{2} \Delta}{\left(\sqrt{X_{+}}+\sqrt{X_{-}}\right)^{2} \sin ^{2} \Delta} \approx 1,
\end{aligned}
$$

then from Eqn. (34)-(37) of [11] we find

$$
\begin{aligned}
\langle\sin \delta\rangle_{+}-\langle\sin \delta\rangle_{-} & =2\left\{\frac{\sqrt{P}+\sqrt{\bar{P}}}{\sqrt{X_{+}}+\sqrt{X_{-}}}\right\}\left\{\frac{\sqrt{X_{+}}\left(\sqrt{X_{+}}-\sqrt{X_{-}}\right)}{Y_{+} \sin \Delta}\right\} \Omega^{-1}, \\
\langle\theta\rangle & =\left\{\frac{\sqrt{P}+\sqrt{\bar{P}}}{\sqrt{X_{+}}+\sqrt{X_{-}}}\right\} \Omega^{-1} \\
\text { and } \quad \theta_{\text {crit }} & =\left\{\frac{Y_{+} \sin \Delta}{\sqrt{X_{+}}\left(\sqrt{X_{+}}-\sqrt{X_{-}}\right)}\right\} \Omega^{-\frac{1}{2}} .
\end{aligned}
$$

These solutions therefore satisfy

$$
\langle\sin \delta\rangle_{+}-\langle\sin \delta\rangle_{-}=2 \Omega^{-\frac{1}{2}}\langle\theta\rangle / \theta_{\text {crit }}
$$

throughout the overlap region. This identity is identical to Eqn. 11] up to small corrections.

This identity is only useful and informative if both $\left|\theta_{i}-\theta_{j}\right|$ and $\left|\sin \delta_{i}-\sin \delta_{j}\right|$ for $(\mathrm{i}, \mathrm{j})=$ $(1,2)$ or $(3,4)$ are small i.e. in the same hierarchy. From the solutions in Ref.[11], one can easily derive that

$$
\left|\theta_{i}-\theta_{j}\right| \leq \beta \theta_{\text {crit }}= \begin{cases}\approx 0 & \mathrm{~T} 2 \mathrm{~K} \\ \leq 0.02 & \mathrm{NO} \nu \mathrm{A}\end{cases}
$$

For $\mathrm{NO} \nu \mathrm{A}$ this restricts the usefulness of our identity to $\sin ^{2} 2 \theta_{13}>10^{-3}$.

The difference between the two values of $\sin \delta$ in the SAME hierarchy from Eqn.(34) and 
(35) of Ref.[11] is bounded by

$$
\begin{aligned}
\left|\sin \delta_{i}-\sin \delta_{j}\right| & \leq \beta=\frac{\left(\sqrt{X_{+}}-\sqrt{X_{-}}\right) \cos \Delta}{\left(\sqrt{X_{+}}+\sqrt{X_{-}}\right) \sin \Delta} \text { for }(\mathrm{i}, \mathrm{j})=(1,2) \text { or }(3,4) \\
& \approx(a L)\left(\Delta^{-1}-\cot \Delta\right) \cot \Delta= \begin{cases}\approx 0 & \mathrm{~T} 2 \mathrm{~K}, \\
\leq 0.1 & \mathrm{NO} \nu \mathrm{A} .\end{cases}
\end{aligned}
$$

for $(\mathrm{i}, \mathrm{j})=(1,2)$ or $(3,4)$.

In conclusion, the identity presented in this paper is accurate, useful and informative for all values of the parameters that can be probed by the proposed experiments T2K and $\mathrm{NO} \nu \mathrm{A}$. For very small values of $\theta_{13}$, beyond the reach of these experiments, there can be significant corrections but here the separation of the $\sin \delta$ 's between the hierarchies is small. 


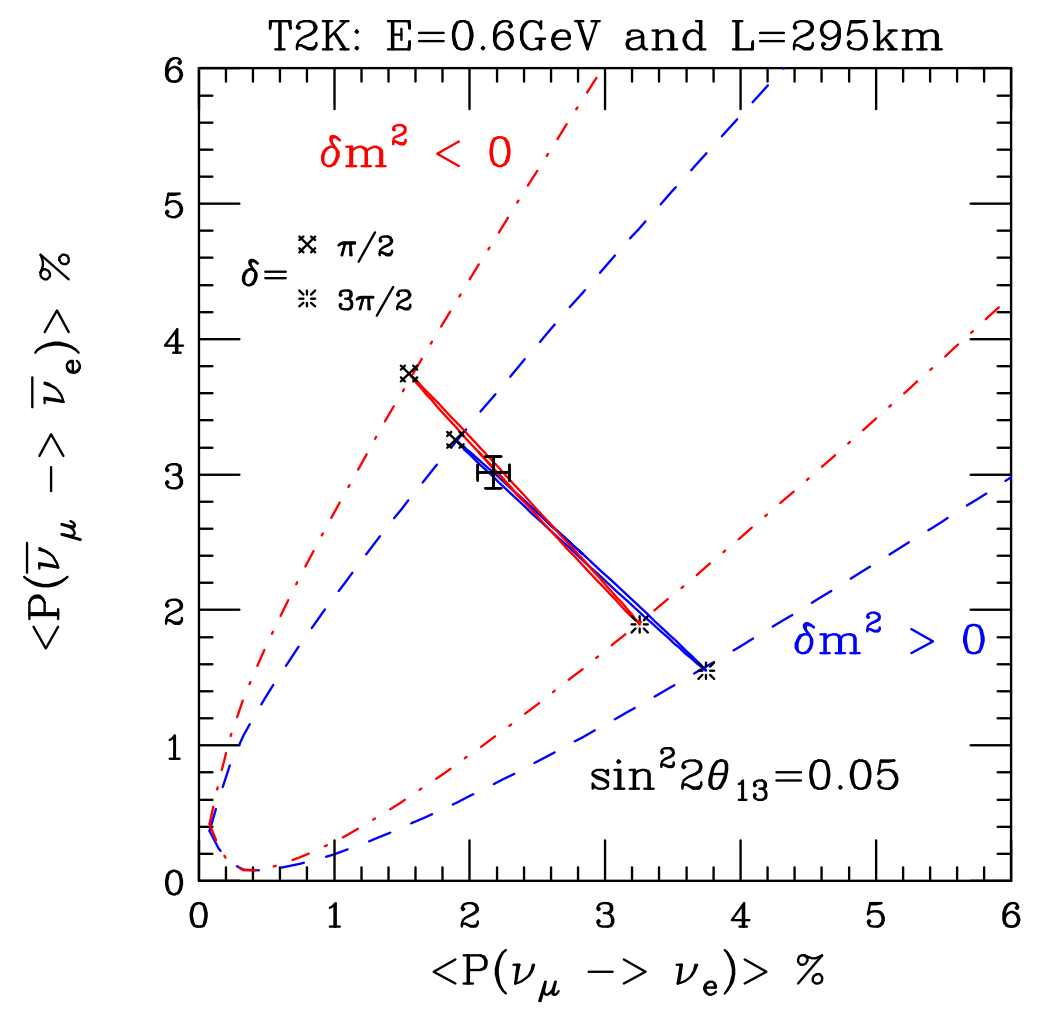

FIG. 1: The bi-probability diagram for T2K showing the allowed regions for both the normal (dashed) and inverted (dotdashed) hierarchies as well as the ellipses for $\sin ^{2} 2 \theta_{13}=0.05$. The large "十" marks the neutrino and anti-neutrino probabilities with the CP phase, $\delta=\pi / 4$, assuming the normal hierarchy. The critical value for this experiment is way off this figure. 


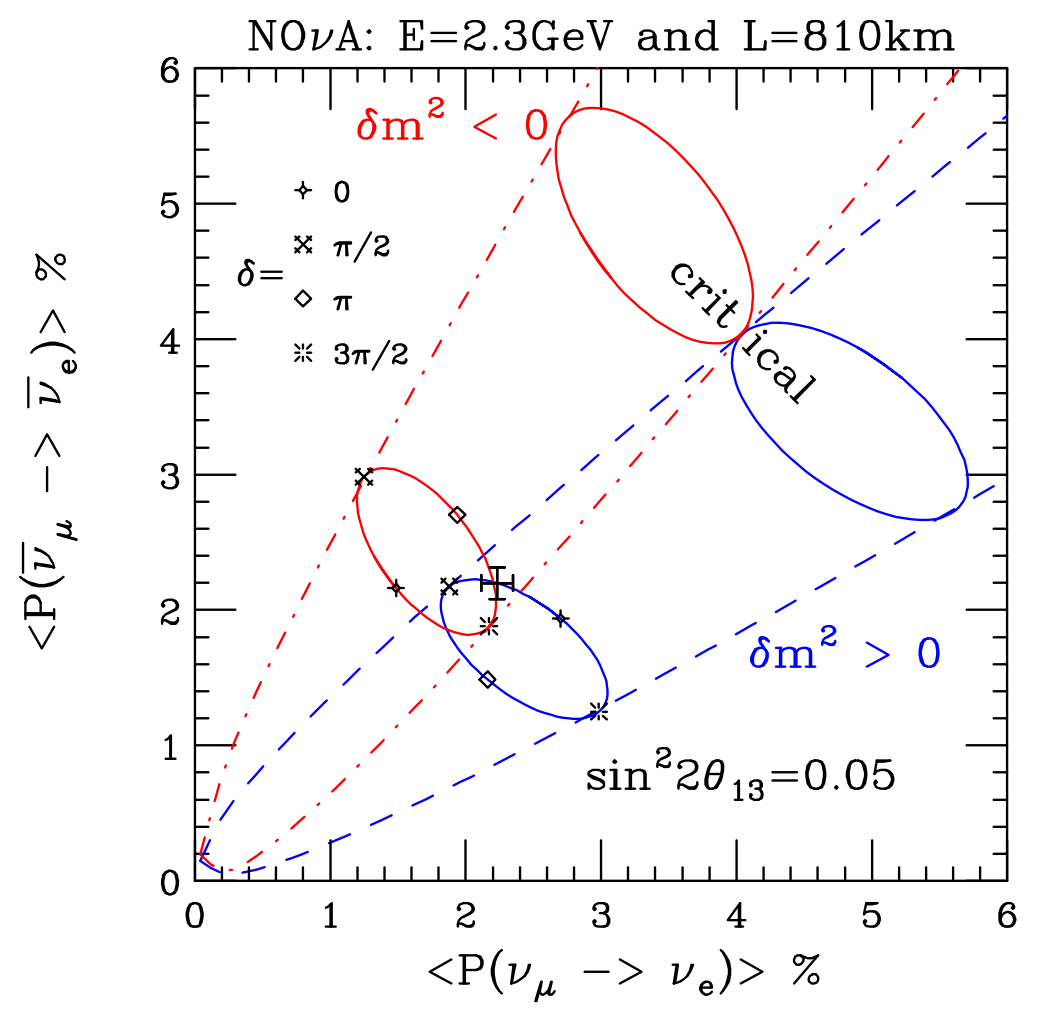

FIG. 2: The bi-probability diagram for $\mathrm{NO} \nu \mathrm{A}$ showing the allowed regions for both the normal (dashed) and inverted (dotdashed) hierarchies as well as the ellipses for $\sin ^{2} 2 \theta_{13}=0.05$. The large "+" marks the neutrino and anti-neutrino probabilities with the CP phase, $\delta=\pi / 4$, assuming the normal hierarchy. The ellipses and point along the diagonal labeled critical correspond to the largest values for which there is overlap between the normal and inverted hierarchies. 


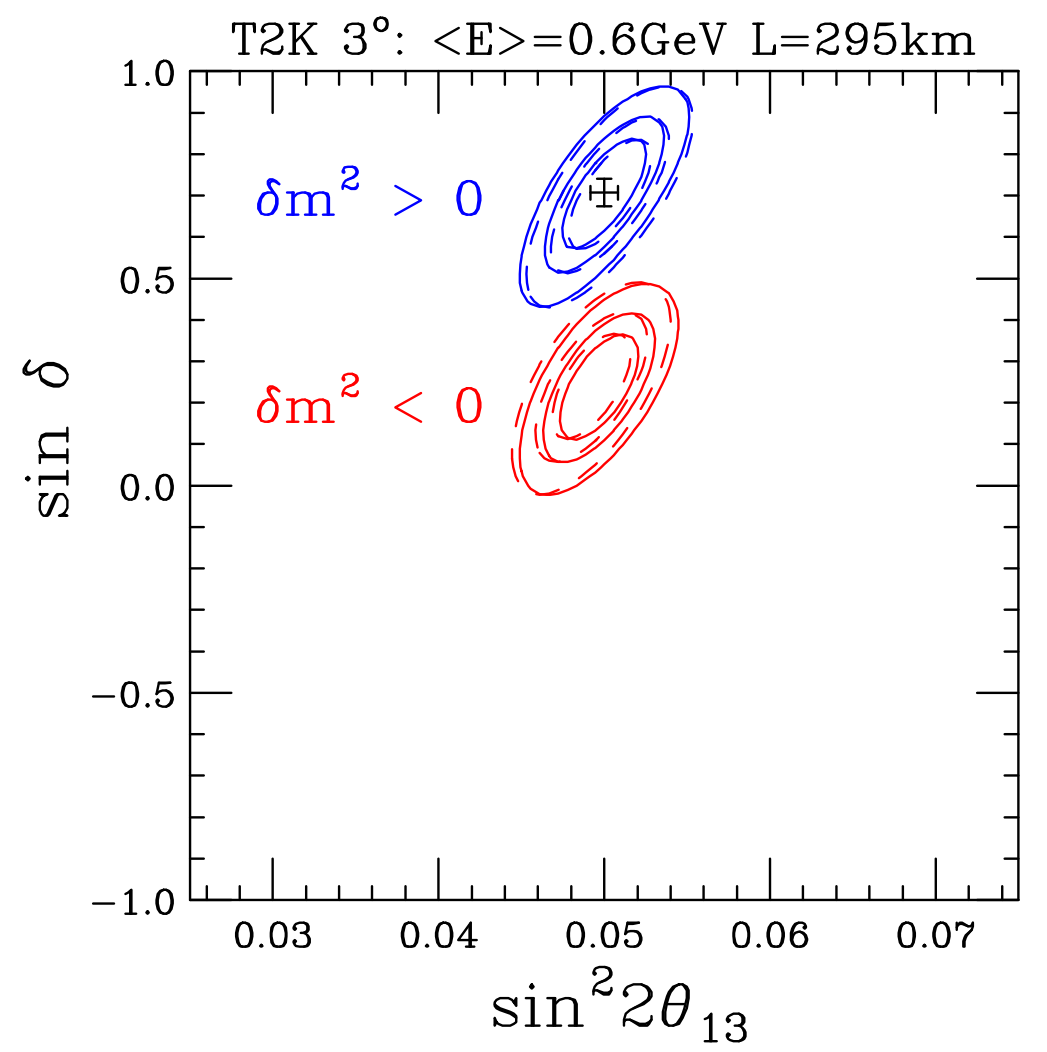

FIG. 3: The allowed regions in the $\sin \delta \mathrm{v} \sin ^{2} 2 \theta_{13}$ plane for $\mathrm{T} 2 \mathrm{~K}$ experiment, assuming the true solution is the normal hierarchy with $\sin ^{2} 2 \theta_{13}=0.05$ and $\delta=\pi / 4$ ("+"). The upper blue (lower red) contours are for the normal (inverted) hierarchy whereas the solid (dashed) contours are for $\cos \delta>0(<0)$. The exposure is 5 years of both neutrino and anti-neutrino running using a 0.75 MW beam at $3^{\circ}$ off-axis and HyperKamiokande $(30 \times 22.5 \mathrm{ktons}$ fiducial mass $)$ as the far detector. The ellipses correspond to 68, 90 and $99 \%$ C.L. contours. If the beam intensity is upgraded to $4 \mathrm{MW}$ but only SuperKamiokande is used as the detector the size of the ellipses is significantly increased. 


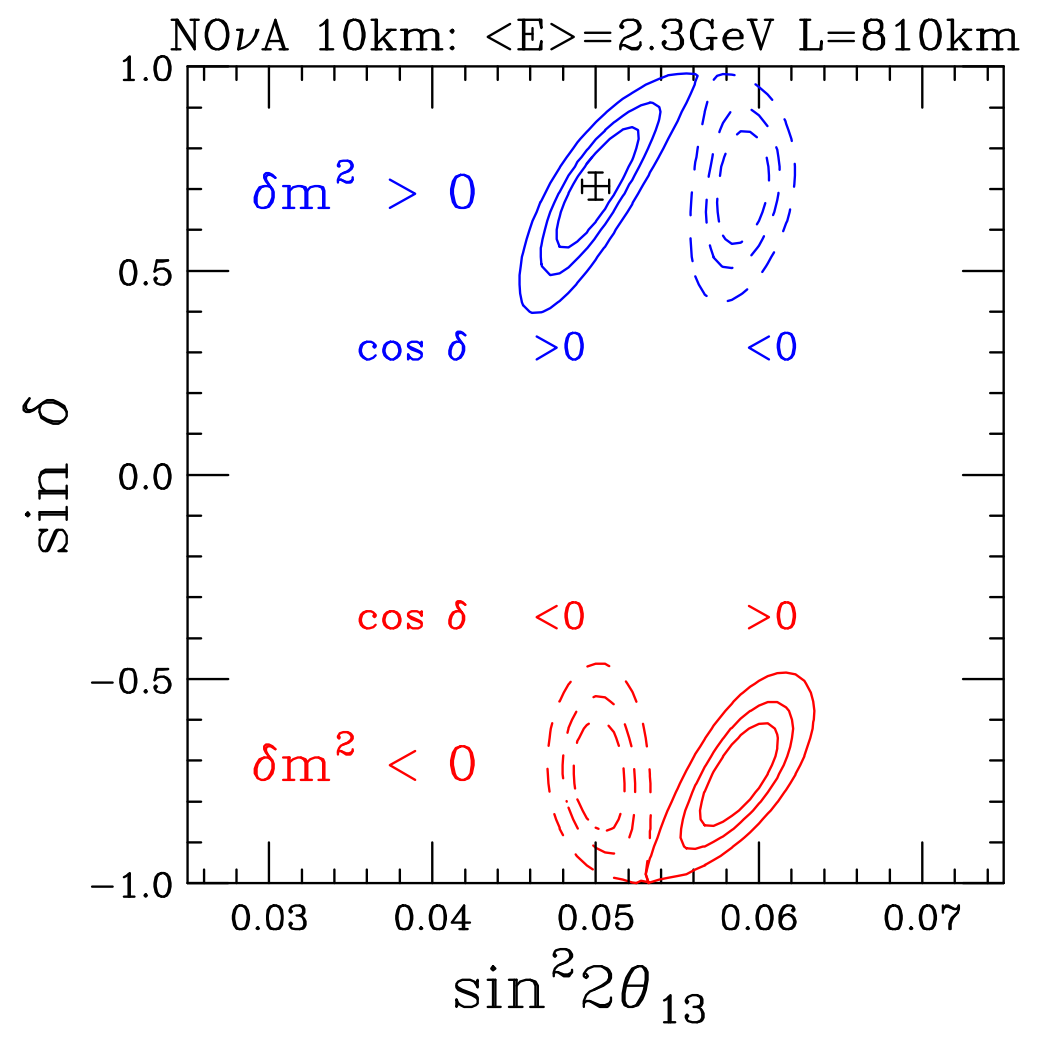

FIG. 4: The allowed regions in the $\sin \delta \mathrm{v} \sin ^{2} 2 \theta_{13}$ plane for the $\mathrm{NO} \nu \mathrm{A}$ experiment, assuming the true solution is the normal hierarchy with $\sin ^{2} 2 \theta_{13}=0.05$ and $\delta=\pi / 4$ ("+"). The upper blue (lower red) contours are for the normal (inverted) hierarchy whereas the solid (dashed) contours are for $\cos \delta>0(<0)$. The exposure is 5 years of both neutrino and anti-neutrino running using a $2 \mathrm{MW}$ beam at $10 \mathrm{~km}$ off-axis and $50 \mathrm{kton}$ low $\mathrm{Z}$ detector. The ellipses correspond to 68, 90 and 99\% C.L. contours. 
[1] H. Minakata, H. Sugiyama, O. Yasuda, K. Inoue and F. Suekane, Phys. Rev. D 68, 033017 (2003); P. Huber, M. Lindner, T. Schwetz and W. Winter, Nucl. Phys. B 665487 (2003); M. H. Shaevitz and J. M. Link, hep-ex/0306031; K. Anderson et al., hep-ex/0402041; O. Yasuda, hep-ph/0403162 F. Ardellier et al., hep-ex/0405032; APS Neutrino Study: Reactor Working Group Report, http://apsreactor.uchicago.edu/report.html.

[2] Y. Itow et al., hep-ex/0106019 http://neutrino.kek.jp/jhfnu/

[3] I. Ambats et al. [NOvA Collaboration], FERMILAB-PROPOSAL-0929; http://www-nova.fnal.gov/

[4] L. Wolfenstein, Phys. Rev. D 17, 2369 (1978); V. D. Barger, K. Whisnant, S. Pakvasa and R. J. N. Phillips, Phys. Rev. D 22, 2718 (1980); S. P. Mikheev and A. Y. Smirnov, Sov. J. Nucl. Phys. 42, 913 (1985); S. J. Parke, Phys. Rev. Lett. 57, 1275 (1986); H. W. Zaglauer and K. H. Schwarzer, Z. Phys. C 40, 273 (1988); M. C. Banuls, G. Barenboim and J. Bernabeu, Phys. Lett. B 513, 391 (2001); M. Freund, M. Lindner, S. T. Petcov and A. Romanino, Nucl. Phys. B 578, 27 (2000).

[5] J. Arafune, M. Koike and J. Sato, Phys. Rev. D 56, 3093 (1997) [Erratum-ibid. D 60, 119905 (1999)]; H. Minakata and H. Nunokawa, Phys. Lett. B 413, 369 (1997); A. Donini, M. B. Gavela, P. Hernandez and S. Rigolin, Nucl. Phys. B 574, 23 (2000).

[6] E. Kearns, Atmospheric neutrino results from SuperKamiokande and T. Nakaya, K2K results, talks given at Neutrino 2004 (Paris), http://neutrino2004.in2p3.fr/

[7] Additional (fake) solutions should arise if $\theta_{23} \neq \pi / 4$, see G. L. Fogli and E. Lisi, Phys. Rev. D 54, 3667 (1996).

[8] J. Burguet-Castell, M. B. Gavela, J. J. Gomez-Cadenas, P. Hernandez and O. Mena, Nucl. Phys. B 608, 301 (2001).

[9] H. Minakata and H. Nunokawa, JHEP 0110, 001 (2001)

[10] V. D. Barger, S. Geer, R. Raja and K. Whisnant, Phys. Rev. D 62 (2000) 013004.

[11] H. Minakata, H. Nunokawa and S. J. Parke, Phys. Rev. D 66 (2002) 093012.

[12] In the following list of references we list some of the previous studies of the parameter degeneracies: J. Burguet-Castell, M. B. Gavela, J. J. Gomez-Cadenas, P. Hernandez and O. Mena, Nucl. Phys. B 646 (2002) 301; A. Donini, D. Meloni and P. Migliozzi, Nucl. Phys. B 646, 
321 (2002); H. Minakata, H. Nunokawa and S. J. Parke, Phys. Lett. B 537, 249 (2002); V. Barger, D. Marfatia and K. Whisnant, Phys. Lett. B 560 (2003) 75; P. Huber, M. Lindner and W. Winter, Nucl. Phys. B 654 (2003) 3; H. Minakata, H. Nunokawa and S. J. Parke, Phys. Rev. D 68 (2003) 013010; hep-ph/0310023; A. Donini, D. Meloni and S. Rigolin, JHEP 0406, 011 (2004); A. Donini, E. Fernandez-Martinez, P. Migliozzi, S. Rigolin and L. Scotto Lavina, hep-ph/0406132

[13] M. Aoki, K. Hagiwara and N. Okamura, Phys. Lett. B 554, 121 (2003). 